\title{
Individual and Mass Behaviour in Large Population Stochastic Wireless Power Control Problems: Centralized and Nash Equilibrium Solutions*
}

\author{
Minyi Huang ${ }^{\dagger}, \quad$ Peter E. Caines ${ }^{\ddagger}, \quad$ and Roland P. Malhamé ${ }^{\S}$
}

\begin{abstract}
We consider uplink power control for lognormal fading channels in the large population case. First, we examine the structure of the control law in a centralized stochastic optimal control setup. We analyze the effect of large populations on the individual control inputs. Next, we split the centralized cost to approach the problem in a game theoretic framework. In this context, we introduce an auxiliary LQG control system and analyze the resulting $\varepsilon=$ Nash equilibrium for the control law; subsequently we generalize the methodology developed for the LQG problem to the wireless power control problem to get an approximation for the collective effect of all other users on a given user. The obtained state aggregation technique leads to highly localized control configurations in contrast to the full state based optimal control strategy.
\end{abstract}

\section{INTRODUCTION}

In wireless communication systems, power control plays a critical role in maintaining an adaptable Quality of Services (QoS) under fading channel conditions and, indeed, power control has recently attracted the research interest of many authors; see [1], [3], [14], [17] and references therein. It is particularly important in CDMA (Code Division Multiple Access) systems in which all users shall the same wideband and act as a source of interference to each other. In a series of papers [7]-[12], power control for lognormal fading channels has been considered as a stochastic control problem, and the optimal control is analyzed by Hamilton-Jacobi-Bellman (HJB) equations. Approximation techniques and numerical methods for computing various suboptimal versions of the optimal control law have been developed in [8], [11], [12]. However, for systems with large populations, there exists the basic limitation of computational complexity associated with this approach. Hence it is desirable to develop new techniques for obtaining simplified yet efficient control laws.

Based on the work mentioned above, this paper makes an attempt to analyze the properties of systems operating in large population conditions. The system includes the lognormal fading channel and a rate based uplink power control model. Our interest is in investigating the feasibility of localized or decentralized control since this may potentially reduce implementational complexity of the control laws. As a first step, we examine the structure of the optimal

\footnotetext{
*Work supported by NCE-MITACS Program 1999-2002 and NSERC Grants 1329-00, 1361-00.

$t: \ddagger$ Department of Electrical \& Computer Engineering,McGill University, 3480 University Street, Montreal, QC, Canada, H3A 2A7. Email: \{mhuang, peterc\}acim. mcgill.ca.

$\S$ Department of Electrical Engineering, École Polytechnique de Montréal, 2900 Edouard Montpetit, Montreal, QC, Canada, H3C 3A7. Email: roland.malhameepolymtl. ca.
}

control law. The feedback control is affine in the system power with a random gain matrix which carries the channel information. Furthermore, the power adjustment rate for each mobile is determined by its own channel state, its own power level and an average effect of all other mobiles. Intuitively, when the population size is large, this average exhibits a statistically stable behaviour with respect to which the action of a specific mobile is negligible. Hence we develop power control methodologies which are less complex than these using the full system state.

As an effective solution toward simplified power control, we may consider a reformulation of the control problem in two aspects. On one hand, we may consider control design by recasting the centralized cost measure into a set of individual user's cost functions; this leads to the game theoretic approach. On the other hand, concerning information pattern of individual user's control inputs, in contrast to the above centralized control structure we may develop decentralized control such that each user utilizes only its local information; this is possible since in the large population scenario (w.r.t the centralized cost or individual costs) the impact of all other users on a given user exhibits a deterministic feature in its evolution. We note that the above techniques concerning the cost type or control information may be combined together to get specific power control formulations.

As a first step, in the control determination of a fixed individual user, we group the effect of all other users into a single term and consider its approximation. This is reasonable due to the special structure of the cost function reflecting the QoS measurement. By this means, we can capture the interaction between the behaviour of any single user and the statistical behaviour of the overall system.

Before proceeding with the analysis for the power control problem, in Section IV we first introduce an auxiliary LQG control system and analyze the individual cost based $\varepsilon$-Nash equilibrium properties for the control law. Subsequently we consider its generalization to the stochastic power control problem in Section V. The method developed for the LQG problem, combined with some reasonable hypotheses, enables us in the power control problem to get an approximation for the collective effect of all the other individuals on any given individual mobile. The procedure has connections with the single user based control design in previous work [11], [12], where we appropriately scaled the total interference generated by all the other mobiles and treated this scaled quantity as a slowly time-varying process. But in the present work, we attempt to capture the dynamics for the evolution 
of the mass formed by the population of users.

We emphasize that the above state aggregation technique for approximately optimal control (with respect to individual costs and the associated mass behaviour) leads to highly localized control configurations, in contrast to the full state based optimal control strategy. Specifically, the control of a particular individual mobile can be formulated in terms of its own channel dynamics, its own state, the aggregated system dynamics and the average of the interference the mobile receives from a mass or collective representing all other users. Finally, in Section VI we make general remarks comparing the centralized cost based optimal control with the individual cost based decentralized control.

\section{The Problem Statement}

Let $x_{i}(t), 1 \leq i \leq n$, denote the attenuation (expressed in $\mathrm{dBs}$ and scaled to the natural logarithm basis) at the instant $t$ of the power of the $i$-th mobile of a network and let $\alpha_{i}(t)=$ $e^{x_{i}(t)}$ denote the actual power attenuation. Based on the work in [4], we model the power attenuation dynamics of $n$ mobile users by

$$
d x_{i}=-a_{i}\left(x_{i}+b_{i}\right) d t+\sigma_{i} d w_{i}, \quad t \geq 0, \quad 1 \leq i \leq n,
$$

where $\left\{w_{i}, 1 \leq i \leq n\right\}$ are $n$ independent standard Wiener processes, and $x_{i}(0), 1 \leq i \leq n$, are mutually independent Gaussian random variables which are also independent of the Wiener processes. In (2.1) $a_{i}, b_{i}, \sigma_{i}>0,1 \leq i \leq n$.

We model the step-wise adjustments [13] of the transmitted power $p_{i}$ (i.e., the uplink power control for the $i$-th mobile) by the so-called rate adjustment model [7]

$$
d p_{i}=u_{i} d t, \quad 1 \leq i \leq n .
$$

We write $x=\left[x_{1}, \cdots, x_{n}\right]^{\tau}, p=\left[p_{1}, \cdots, p_{n}\right]^{\tau}, u=$ $\left[u_{1}, \cdots, u_{n}\right]^{\tau}$. In a CDMA context, the signal-to-interference ratio (SIR) for the users achieved after matched filtering is given by $\Gamma_{i}=\frac{\widehat{p}_{i}}{\sum_{k \neq i} \beta_{k, i} \hat{p}_{k}+\eta}, 1 \leq i \leq n$, where $\widehat{p}_{i}$ denotes the received power at the based station for user $i, \beta_{k, i}=\left(\mathbf{s}_{k}^{r} \mathbf{s}_{i}\right)^{2}, k \neq j$, is the squared crosscorrelation between the (normalized) signature sequences $\mathbf{s}_{k}, \mathbf{s}_{i}$ of users $k$, $i$, respectively, and $\eta$ is the constant background noise intensity. Denote the dimension (i.e., the spreading gain) of $\mathbf{s}_{i}$ by $n_{s}$. Following [15], [16], [17], we consider the mobile system in the context of a large number of users and make the standard assumption that $\frac{n}{n_{s}}=\alpha>0$ as $n \rightarrow \infty$, i.e., the signature length $n_{s}$ increases in proportion to the user population, which is necessary to suppress the interuser interference so as to accommodate an increasing number of users. Here $\alpha$ is called the number of users per degree of freedom. By appropriately choosing random signature sequences of length $n_{s}$, one can have $\beta_{k, i} \approx \frac{1}{n_{s}}=\frac{\alpha}{n}$ [15], [17]. For simplicity, here we take $\beta_{k, i}=\frac{1}{n}$ for all $1 \leq k \neq i \leq n$. Moreover, we wish $\Gamma_{i}$ to be staying around a target SIR level $\gamma_{i} \in(0,1)$, i.e.,

$$
\Gamma_{i}=\frac{\widehat{p}_{i}}{\frac{1}{n} \sum_{k \neq i} \widehat{p}_{k}+\eta} \approx \gamma_{i}, \quad 1 \leq i \leq n .
$$

Under the condition of lognormal fading we have $\widehat{p}_{i}=$ $e^{x_{i}} p_{i}, 1 \leq i \leq n$, where $x_{i}$ is described by (2.1).

Following [8], [11] and taking into account the SIR requirement $(2.3)$, we introduce the following modified loss function:

$$
E \int_{0}^{\infty} e^{-\rho t}\left\{\sum_{i=1}^{n}\left[e^{x_{i}} p_{i}-\gamma_{i} N_{i}\right]^{2}+u^{\tau} R u\right\} d t .
$$

where $N_{i}=\frac{1}{n} \sum_{j \neq i}^{n} e^{x_{j}} p_{j}+\eta, \rho>0$ and $R>0$ is a weight matrix. For simplicity we take $R=\operatorname{Diag}\left(r_{i}\right)_{i=1}^{n}>0$. In the above integrand the term $u^{\tau} R u$ is used to penalize abrupt change of powers since in practical systems power adjustment is exercised in a cautious manner. After subtracting the constant component from the integrand in (2.4) we get the cost function to be employed:

$$
J(u)=E \int_{0}^{\infty} e^{-\rho t}\left[p^{\tau} C(x) p+2 D^{\tau}(x) p+u^{\tau} R u\right] d t
$$

where $C(x), D(x)$ are $n \times n$ positive definite matrix, $n \times 1$ vector, respectively, which are determined from (2.4). Write $l(x, p, u)=p^{\tau} C(x) p+2 D^{\tau}(x) p+u^{\tau} R u$.

To facilitate further analysis, we set $f_{i}(x)=-a_{i}\left(x_{i}+\right.$ $\left.b_{i}\right), 1 \leq i \leq n, H=\operatorname{Diag}\left(\sigma_{i}\right)_{i=1}^{n}$ and $z^{\tau}=\left(x^{\tau}, p^{\tau}\right), \psi^{\tau}=$ $\left(f^{\tau}, u^{\tau}\right), G^{\tau}=\left(H, 0_{n \times n}\right)$. We write (2.1) and (2.2) in the vector form

$$
d z=\psi d t+G d w, \quad t \geq 0,
$$

where $w$ is an $n \times 1$ standard Wiener process determined by (2.1). We will denote the state variable by $(x, p)$ or $z$. Define the admissible control set $\mathcal{U}=\left\{u \mid u\right.$ is adapted to $\sigma\left(x_{s}, s \leq\right.$ $t)$, and $\left.E \int_{0}^{\infty} e^{-\rho t}\left|u_{t}\right|^{2} d t<\infty\right\}$. We assume that $\left.p\right|_{t=0}$ is a deterministic. We note that certain controls from $\mathcal{U}$ may result in an infinite cost due to the presence of the $e^{x_{i}}$ process, $1 \leq i \leq n$. However the optimal control problem is well defined under the admissible control set $\mathcal{U}$. Finally, the cost associated with (2.6) and a control $u$ is $J(x, p, u)=E\left[\int_{0}^{\infty} e^{-\rho t} l\left(x_{t}, p_{t}, u_{t}\right) d t \mid x_{0}=x, p_{0}=p\right]$, where $(x, p)$ is the initial state; further we set the value function $v(x, p)=\inf _{u \in \mathcal{U}} J(x, p, u)$.

\section{The Value Function and HJB Equation}

Formally applying dynamic programming, we may write the HJB equation for the value function $v$ as

$$
\begin{gathered}
\rho v=-\sum_{i=1}^{n} a_{i}\left(x_{i}+b_{i}\right) \frac{\partial v}{\partial x_{i}}+\frac{1}{2} \sum_{i=1}^{n} \sigma_{i}^{2} \frac{\partial^{2} v}{\partial x_{i}{ }^{2}}-\frac{1}{4} v_{p}^{\tau} R^{-1} v_{p} \\
+p^{\tau} C(x) p+2 D^{\tau}(x) p
\end{gathered}
$$

Proposition 3.1: [8] The value function $v$ is a classical solution to (3.1) and can be written as

$$
v(x, p)=p^{\tau} K(x) p+2 p^{\tau} S(x)+q(x)
$$


where $K(x)=K^{\tau}(x), S(x), q(x)$ are continuous in $x$, and are all of order $O\left(1+\sum_{i=1}^{n} e^{2 x_{i}}\right)$.

In general, additional growth conditions as above are required in order to determine the value function by the HJB equation when there is no boundary condition (see e.g. [5]). Substituting (3.2) into (3.1) and comparing powers of $p$, we obtain the partial differential equation system

$$
\begin{aligned}
& \rho K=\frac{1}{2} \sum_{k=1}^{n} \sigma_{k}^{2} \frac{\partial^{2} K}{\partial x_{k}{ }^{2}}+\sum_{k=1}^{n} f_{k} \frac{\partial K}{\partial x_{k}}-K R^{-1} K+C, \\
& \rho S=\frac{1}{2} \sum_{k=1}^{n} \sigma_{k}^{2} \frac{\partial^{2} S}{\partial x_{k}{ }^{2}}+\sum_{k=1}^{n} f_{k} \frac{\partial S}{\partial x_{k}}-K R^{-1} S+D, \\
& \rho q=\frac{1}{2} \sum_{k=1}^{n} \sigma_{k}^{2} \frac{\partial^{2} q}{\partial x_{k}{ }^{2}}+f^{\tau} \frac{\partial q}{\partial x}-S^{\tau} R^{-1} S,
\end{aligned}
$$

where we shall refer to (3.3) as the Riccati equation. The optimal control law for the $n$ users is given by

$$
u=\left[u_{1}, \cdots, u_{n}\right]^{\tau}=-R^{-1}[K(x) p+S(x)],
$$

and for user $i$ the control is

$$
u_{i}=-\frac{1}{r_{i}} K_{i i}(x) p_{i}-\frac{1}{r_{i}} \sum_{j \neq i} K_{i j}(x) p_{j}-\frac{1}{r_{i}} S_{i}(x) .
$$

It is seen from (3.7) that for user $i$, the control relies on its own current power level and a weighted sum of other users' powers. Since all the coefficients involved in this individual control law depend on the attenuations of all users, this optimal control law is highly centralized and hence unrealistic for a practical implementation for systems with large populations. However, the randomness associated with the second term in (3.7) should be small due to the scaling effect of $K_{i j}(x), j \neq i$, and the superposition of many small terms, and hence we may consider the average effect of the other users' powers on a given user. This further suggests we consider developing state aggregation techniques (to approximate the mass behavior) from the level of both performance measure and control determination for individual users. We note that in the centralized LQG control case, under symmetric dynamics for individuals a decomposition of each individual's control into an individual term involving its own state and a deterministic term for the mass may be explicitly obtained when the population size goes to infinity [6]. tions:

To simplify our analysis, we make the symmetry assump-

C1) All users have i.i.d. dynamics, i.e., $a_{i}=a, b_{i}=b$, $\sigma_{i}=\sigma, 1 \leq i \leq n$;

C2) All users have equal SIR requirements, i.e., $\gamma_{i}=\gamma$, $1 \leq i \leq n$, and in addition, $R=r I_{n}$.

Before further analysis of the control for individual users in Section V, we examine in below linear systems with a cost which can be regarded as the limiting version of the cost (2.5) by freezing the random attenuation $x$ as a constant. We will develop the basic idea for state aggregation via this auxiliary linear model, and then generalize this method to the nonlinear power control problem of Sections II-III in Section V.

\section{DYNAmICALly INDEPENDENT AND Cost CouPled LARGE POPULATION LQG SYSTEMS}

Suppose in a linear stochastic system, the state of each of the $n$ individuals or players is described by

$$
d z_{i}=\left(a z_{i}+b u_{i}\right) d t+\sigma d w_{i}, \quad t \geq 0, \quad 1 \leq i \leq n,
$$

where $\left\{w_{i}, 1 \leq i \leq n\right\}$ denotes $n$ independent standard scalar Wiener processes and $b \neq 0$. The initial state $z_{i}(0)$, $1 \leq i \leq n$, is independent of the Wiener processes and $E z_{i}^{2}(0)<\infty$. The $n$ individuals interact with each other through a global cost function

$$
\begin{aligned}
J & =J\left(u_{1}, v_{1} ; u_{2}, v_{2} ; \cdots ; u_{n}, v_{n}\right)=\sum_{i=1}^{n} J_{i}\left(u_{i}, v_{i}\right) \\
& \triangleq E \int_{0}^{\infty} \sum_{i=1}^{n} e^{-\rho t}\left[\left(z_{i}-v_{i}\right)^{2}+r u_{i}^{2}\right] d s,
\end{aligned}
$$

and in particular we set in the cost-coupled case $v_{i}=$ $\gamma\left(\frac{1}{n} \sum_{k \neq i}^{n} z_{k}+\eta\right)$. Notice that in this Section $z_{i}$ is described by the general dynamics (4.1). In order to avoid introducing too many new variables and parameters, we ask the reader to consider that the notation $\left(a, b, \sigma, u_{i}, z_{i}\right)$ in this Section is independent of Sections II-III. As in the previous Sections, here we also assume $\rho, r, \gamma, \sigma, \eta>0$.

\section{A. Competitive Behaviour: State Aggregation and Tracking for LQG Systems}

We denote the mass driven version of the individual cost for the $i$-th player as $J_{i}\left(u_{i}, \gamma\left(\frac{1}{n} \sum_{k \neq i}^{n} z_{k}+\eta\right)\right)$. Furthermore, for large $n$, assume $z_{-i}^{*} \triangleq \gamma\left(\frac{1}{n} \sum_{k \neq i}^{n} z_{k}+\eta\right)$ is approximated by a deterministic function $z^{*}(t)$. We construct the $z^{*}$ driven version of the individual cost for the $i$-th player

$$
J_{i}\left(u_{i}, z^{*}\right)=E \int_{0}^{\infty} e^{-\rho t}\left\{\left[z_{i}-z^{*}(t)\right]^{2}+r u_{i}^{2}\right\} d s,
$$

We note that for large $n$, it is reasonable to use a single $z^{*}(t)$ to approximate all $z_{-i}^{*}, 1 \leq i \leq n$. Let $C_{b}[0, \infty)$ be the set of bounded continuous functions on $[0, \infty)$.

Proposition 4.1: Assuming $\Pi>0$ and $s \in C_{b}[0, \infty)$ are determined by the following scalar equations

$$
\begin{aligned}
& \rho \Pi=2 a \Pi-\frac{b^{2}}{r} \Pi^{2}+1, \\
& \rho s=\frac{d s}{d t}+a s-\frac{b^{2}}{r} \Pi s-z^{*}(t),
\end{aligned}
$$

where $z^{*} \in C_{b}[0, \infty)$, then $u_{i}=-\frac{b}{r}\left(\Pi z_{i}+s\right)$ is the optimal control minimizing $J_{i}\left(u_{i}, z^{*}\right)$.

The proof can be done by an algebraic approach as in [2] and the details are omitted here. We denote

$$
\beta_{1}=-a+\frac{b^{2}}{r} \Pi, \quad \beta_{2}=\rho-a+\frac{b^{2}}{r} \Pi
$$


By well known results for Riccati equations, we have $a-$ $\frac{b^{2}}{r} \Pi-\frac{\rho}{2}<0$ and hence $\beta_{2}>0$. In fact, $s \in C_{b}[0, \infty)$ may be uniquely expressed as $s(t)=-e^{\beta_{2} t} \int_{t}^{\infty} e^{-\beta_{2} \tau} z^{*}(\tau) d \tau \rightarrow$ $-\frac{z^{*}(\infty)}{\beta_{2}}$, as $t \rightarrow \infty[6]$. Unbounded solutions for $s$ are excluded for our problem.

With control $u_{i}$ given in Proposition 4.1, the closed loop for the $i$-th player is

$$
d z_{i}=\left(a-\frac{b^{2}}{r} \Pi\right) z_{i} d t-\frac{b^{2}}{r} s d t+\sigma d w_{i} .
$$

Denoting $\bar{z}_{i}(t)=E z_{i}(t)$ and taking expectation on both sides of (4.7) yields $\frac{d \bar{z}_{i}}{d t}=\left(a-\frac{b^{2}}{r} \Pi\right) \bar{z}_{i}-\frac{b^{2}}{r} s$, where $\left.\bar{z}_{i}\right|_{t=0}=E z_{i}(0)$. We further define the population mean $\bar{z} \triangleq \frac{1}{n} \sum_{i=1}^{n} \bar{z}_{i}$; then clearly $\bar{z}$ evolves according to the same equation as $z_{i}$, i.e.,

$$
\frac{d \bar{z}}{d t}=\left(a-\frac{b^{2}}{r} \Pi\right) \bar{z}-\frac{b^{2}}{r} s,
$$

where $\left.\bar{z}\right|_{t=0}=\frac{1}{n} \sum_{i=1}^{n} E z_{i}(0)$. Here one naturally comes up with the important issue of relating the deterministic process $z^{*}$ to the collective effect of the population. Since we wish to have $z^{*}(t) \approx z_{-i}^{*}=\gamma\left(\frac{1}{n} \sum_{k \neq i}^{n} z_{k}+\eta\right)$ for large $n$, it is reasonable to express $z^{*}$ in terms of the population mean $\bar{z}$, i.e.,

$$
z^{*}(t)=\gamma(\bar{z}(t)+\eta), \quad t \geq 0 .
$$

Combining (4.5), (4.8) and (4.9) together and setting the derivatives as zero, we write a set of steady state equations as follows

$$
\left\{\begin{array}{l}
\gamma \bar{z}_{\infty}-z_{\infty}^{*}=-\gamma \eta, \\
z_{\infty}^{*}-\left(a-\frac{b^{2}}{r} \Pi-\rho\right) s_{\infty}=0, \\
\left(a-\frac{b^{2}}{r} \Pi\right) \bar{z}_{\infty}-\frac{b^{2}}{r} s_{\infty}=0 .
\end{array}\right.
$$

Example 4.2: For the system $a=1, b=1, \sigma=$ $0.3, \rho=0.5, \gamma=0.6, r=0.1, \eta=0.25$, we get $\Pi=0.4, \quad\left(\bar{z}_{\infty}, z_{\infty}^{*}, s_{\infty}\right)=(0.333333,0.35,-0.1)$.

We make the following key assumptions for this Section:

C3) $\beta_{1}>0$, and $\frac{M}{\beta_{1} \beta_{2}}<1$, where $M=\frac{b^{2} \gamma}{r}$, and $\beta_{1}, \beta_{2}$ are determined by (4.6).

C4) $z_{i}(0), 1 \leq i \leq n$, are mutually independent and $\sup _{i} E z_{i}^{2}(0) \leq C$, for $C$ independent of $n$.

It can be verified that $\mathbf{C 3}$ ) holds for Example 4.2. Under C3), (4.10) is a nonsingular linear equation and has a unique solution $\left(\bar{z}_{\infty}, z_{\infty}^{*}, s_{\infty}\right)$.

Eliminating $s$ in (4.8) by (4.5) and (4.9), we get the equation for the population mean

$$
\frac{d \bar{z}}{d t}=-\beta_{1} \bar{z}+\frac{b^{2} \gamma}{r} \int_{t}^{\infty} e^{\beta_{2}(t-\tau)} \bar{z}(\tau) d \tau+\frac{b^{2} \gamma \eta}{r \beta_{2}} .
$$

Theorem 4.3: Under C3), the integral-differential equation (4.11) with any initial condition $\bar{z}_{0}$ and the terminal condition $\lim _{t \rightarrow \infty} \bar{z}(t)=\bar{z}_{\infty}$ admits a unique solution.

We note that under C3), an explicit bounded solution for the set of equations (4.5), (4.8) and (4.9) may be obtained [6].

\section{B. Decentralized LQG $\varepsilon-N a s h$ Equilibria}

In the current context we give the definition of $\varepsilon$-Nash equilibrium.

Definition 4.4: A set of controls $u_{k} \in \mathcal{U}_{k}, 1 \leq k \leq n$, where $\mathcal{U}_{k}$ is a specified class of measurable functions of the state processes $z_{1}(\cdot), \cdots, z_{n}(\cdot)$ such that the resulting $v_{k}$ is adapted to some subfiltration of the underlying Brownian motion, is called an $\varepsilon-N a s h$ equilibrium with respect to the costs $J_{k}\left(u_{k}, v_{k}\right), 1 \leq k \leq n$, if there exists $\varepsilon \geq 0$ such that for each $1 \leq i \leq n$,

$$
\begin{aligned}
& J_{i}\left(u_{i}, v_{i}\left(u_{1}, \cdots, u_{i-1}, u_{i+1} \cdots, u_{n}\right)\right) \\
\leq & J_{i}\left(u_{i}^{\prime}, v_{i}\left(u_{1}, \cdots, u_{i-1}, u_{i+1}, \cdots, u_{n}\right)\right)+\varepsilon
\end{aligned}
$$

when any alternative $u_{i}^{\prime} \in \mathcal{U}_{i}$ is applied by the $i$-th player. $\square$ In Definition 4.4, when $\varepsilon=0$, we retrieve the usual Nash Equilibrium.

Let $u_{k}^{0}$ be the optimal tracking based control law for the $k$-th player, i.e., $u_{k}^{0}=-\frac{b}{r}\left(\Pi z_{k}+s\right)$ where $s$ and $z^{*}$ are derived from (4.5), (4.8) and (4.9). We recall that the initial condition of $\bar{z}$ is take as $\frac{1}{n} \sum_{k=1}^{n} E z_{k}(0)$. Furthermore, let

$$
\begin{aligned}
& J_{i}\left(u_{i}, v_{i}\left(u_{1}^{0}, \cdots, u_{i-1}^{0}, u_{i+1}^{0} \cdots, u_{n}^{0}\right)\right) \\
\triangleq & E \int_{0}^{\infty} e^{-\rho t}\left\{\left[z_{i}\left(u_{i}\right)-\gamma\left(\frac{1}{n} \sum_{k \neq i}^{n} z_{k}\left(u_{k}^{0}\right)+\eta\right)\right]^{2}+r u_{i}^{2}\right\} d t,
\end{aligned}
$$

denote the mass driven version of the $i$-th individual cost where $z_{k}\left(u_{k}^{0}\right)=z_{k}\left(u_{k}^{0}\left(z_{k}, z^{*}\right)\right)$, and in particular,

$$
\begin{aligned}
& J_{i}\left(u_{i}^{0}, v_{i}\left(u_{1}^{0}, \cdots, u_{i-1}^{0}, u_{i+1}^{0} \cdots, u_{n}^{0}\right)\right) \\
= & \left.J_{i}\left(u_{i}, v_{i}\left(u_{1}^{0}, \cdots, u_{i-1}^{0}, u_{i+1}^{0} \cdots, u_{n}^{0}\right)\right)\right|_{u_{i}=u_{i}^{0}} .
\end{aligned}
$$

Denote $\sigma_{0}^{2}=\sup _{i} E\left[z_{i}(0)-E z_{i}(0)\right]^{2}, \sigma_{0} \geq 0$.

Theorem 4.5: Under (C3)-C4) we have

$$
\begin{aligned}
& \left|J_{i}\left(u_{i}^{0}, \gamma\left(\frac{1}{n} \sum_{k \neq i}^{n} z_{k}\left(u_{k}^{0}\right)+\eta\right)\right)-J_{i}\left(u_{i}^{0}, z^{*}\right)\right| \\
= & O\left(\frac{\sigma+\sigma_{0}}{\sqrt{n}}+\frac{1}{n}\right),
\end{aligned}
$$

where $u_{k}^{0}$ is the optimal tracking based control law.

Theorem 4.6: Under C3)-C4) the set of controls $u_{i}^{0}, 1 \leq$ $i \leq n$, for the $n$ players is an $\varepsilon$-Nash equilibrium with respect to the mass driven version of the individual costs $J_{i}\left(u_{i}, \gamma\left(\frac{1}{n} \sum_{k \neq i}^{n} z_{i}\left(u_{k}\right)+\eta\right)\right), 1 \leq i \leq n$, where $\varepsilon=$ $O\left(\frac{\sigma+\sigma_{0}}{\sqrt{n}}+\frac{1}{n}\right)$. More specifically, for any $i$, we have

$$
\begin{aligned}
& J_{i}\left(u_{i}^{0}, \gamma\left(\frac{1}{n} \sum_{k \neq i}^{n} z_{k}\left(u_{k}^{0}\right)+\eta\right)\right)-O\left(\frac{\sigma+\sigma_{0}}{\sqrt{n}}+\frac{1}{n}\right) \\
& \leq \inf _{u_{i}} J_{i}\left(u_{i}, \gamma\left(\frac{1}{n} \sum_{k \neq i}^{n} z_{k}\left(u_{k}^{0}\right)+\eta\right)\right) \\
& \leq J_{i}\left(u_{i}^{0}, \gamma\left(\frac{1}{n} \sum_{k \neq i}^{n} z_{k}\left(u_{k}^{0}\right)+\eta\right)\right)
\end{aligned}
$$

where $u_{i}$ depends on $\left(t, z_{1}, \cdots, z_{n}\right)$. 


\section{A Cost Gap between Centralized Optimal Control and Decentralized Control}

For the global cost $J$ given by (4.2) with $v_{i}=$ $\gamma\left(\frac{1}{n} \sum_{k \neq i}^{n} z_{k}+\eta\right)$, one can compute the optimal cost with centralized information (i.e., each $u_{i}$ depends on $z_{1}, \cdots, z_{n}$ ) by the standard algebraic Riccati equation approach. Here we assume $z_{i}(0)=0$, for $1 \leq i \leq n$. Denote $\left.v(0) \triangleq \inf J\right|_{z_{i}(0)=0,1 \leq i \leq n}=\left.\inf \left(\sum_{i=1}^{n .} J_{i}\right)\right|_{z_{i}(0)=0,1 \leq i \leq n}$. Let $\bar{v}_{n}(0)=\frac{v(0)}{n}$ and $\bar{v}(0)=\lim _{n \rightarrow \infty} \bar{v}_{n}(0) \cdot \bar{v}(0)$ may be interpreted as the optimal cost incurred by each individual in the large population limit.

On the other hand, for (4.3) we denote $v_{\text {ind }}(0)=$ $\inf _{u_{i}} J_{i}\left(u_{i}, z^{*}\right)$, where $z^{*}$ is determined by (4.5), (4.8) and (4.9) and we take initial condition $z_{i}(0)=0$. Then we have

$$
\left|\bar{v}(0)-v_{\text {ind }}(0)\right|=O\left(\gamma^{2}\right)
$$

see [6] for details. The cost gap is displayed in Fig. 1 below. The plot is obtained from a family of systems specified by: $a=b=1, \sigma^{2}=0.09, \rho=0.5, r=0.1, \eta=0.25$ and $\gamma \in[0,0.6]$.

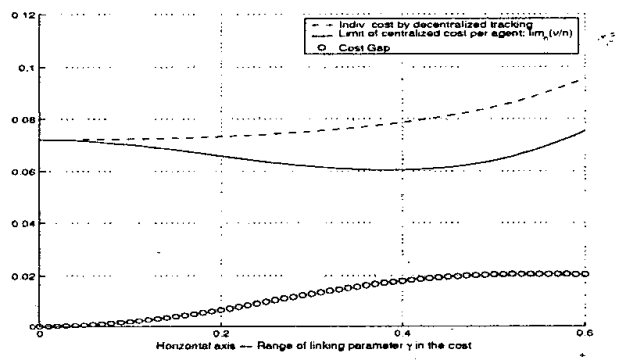

Fig. 1. Top: individual tracking based $\operatorname{cost} v_{i n d}(0)$; Middle: scaled global cost $\bar{v}(0)$; Bottom: the cost gap $\left|\bar{v}(0)-v_{\text {ind }}(0)\right|$.

\section{Decentralized Large Population Wireless POWER CONTROL PROBLEMS}

We now approximate the wireless power control problem of Sections 2-3 in the large population case by a tracking problem together with an exogenous random process $x$ as described by (2.1). In this Section we assume the assumptions C1) and C2) hold. We shall establish a large population $\varepsilon$-Nash equilibrium result by extending the method in Section IV-B. The notation used in this Section is consistent with Sections II-III, and some notation as well as $\varepsilon$-Nash equilibria of Section IV will be extended to the power control context. First, we set the individual cost for the $i$-th mobile with respect to the mass as

$$
\begin{aligned}
& J_{i}\left(u_{i}, \gamma\left(\frac{1}{n} \sum_{k \neq i}^{n} e^{x_{k}} p_{k}+\eta\right)\right) \triangleq \\
& E \int_{0}^{\infty} e^{-\rho t}\left\{\left[e^{x_{i}} p_{i}-\gamma\left(\frac{1}{n} \sum_{k \neq i}^{n} e^{x_{k}} p_{k}+\eta\right)\right]^{2}+r u_{i}^{2}\right\} d t
\end{aligned}
$$

i.e., the $i$-th component in the centralized cost function in Section II. We define the $i$-th individual cost with respect to a deterministic function $z^{*} \in C_{b}[0, \infty)$ as

$$
J_{i}\left(u_{i}, z^{*}\right) \triangleq E \int_{0}^{\infty} e^{-\rho t}\left\{\left[e^{x_{i}} p_{i}-z^{*}(t)\right]^{2}+r u_{i}^{2}\right\} d t
$$

When the individual costs $J_{i}\left(u_{i}, z^{*}\right)$ are employed, assuming sufficient smoothness of the optimal costs

$v\left(t, x_{i}\right) \triangleq \inf _{u_{i}} E\left[\int_{t}^{\infty} e^{-\rho \tau}\left\{\left[e^{x_{i}(\tau)} p_{i}-z^{*}\right]^{2}+r u_{i}^{2}\right\} d \tau \mid x_{i}\right]$,

where $t \geq 0$, we can write the equation system

$$
\begin{aligned}
& \rho K\left(x_{i}\right)=\frac{\sigma^{2}}{2} \frac{\partial^{2} K}{\partial x_{i}{ }^{2}}-a\left(x_{i}+b\right) \frac{\partial K}{\partial x_{i}}-\frac{1}{r} K^{2}+e^{2 x_{i}}, \quad \text { (5.3) } \\
& \rho s\left(t, x_{i}\right)=\frac{\partial s}{\partial t}+\frac{\sigma^{2}}{2} \frac{\partial^{2} s}{\partial x_{i}{ }^{2}}-a\left(x_{i}+b\right) \frac{\partial s}{\partial x_{i}}-\frac{K s}{r}-z^{*} e^{x_{i}}
\end{aligned}
$$

with classical solutions such that $K\left(x_{i}\right)=O\left(1+e^{2 x_{i}}\right)$ and $s\left(t, x_{i}\right)=O\left(1+e^{x_{i}}\right)$ uniformly with respect to $t \in[0, \infty)$. Then by an argument employing a verification theorem [5] one can show that the optimal control law for the $i$-th user minimizing $J_{i}\left(u_{i}, z^{*}\right)$ is

$$
u_{i}=-\frac{1}{r}\left[K\left(x_{i}\right) p_{i}+s\left(t, x_{i}\right)\right]
$$

and hence we have the optimal closed loop for $p_{i}$ as

$$
d p_{i}=-\frac{1}{r}\left[K\left(x_{i}\right) p_{i}+s\left(t, x_{i}\right)\right] d t .
$$

As in the pure LQG case analyzed in Section IV-A, here we also have the issue of determining the function $z^{*}$ which is to be tracked by individual players. With the original SIR based cost function in mind, we consider taking $z^{*} \approx$ $\gamma\left(\frac{1}{n} \sum_{k \neq i} e^{x_{k}} p_{k}+\eta\right)$ for large $n$. We recall that any pair of $x_{i}, x_{k}, i \neq k$, are independent of each other. To simplify our analysis further, we shall assume that each $x_{i}$ has initial condition $\left.x_{i}\right|_{t=0}$ such that $x_{i}$ is a stationary Gaussian process. We also assume that all mobile users have identical deterministic initial condition $\left.p_{i}\right|_{t=0}$. The extension to more general initial conditions for the attenuations and powers appears to be straightforward. Under the above assumptions, we take

$$
z^{*}(t)=\gamma\left(E e^{x_{i}} p_{i}+\eta\right)
$$

where the right hand side only depends on time $t$ and the initial power $p_{0}$ after the feedback is determined by (5.5).

Theorem 5.1: If there exists $\left(K\left(x_{i}\right), s\left(x_{i}\right), z^{*}(t)\right)$ satisfying the equation system (5.3), (5.4), (5.6) and (5.7) such that $z^{*} \in C_{b}[0, \infty), K \in C^{2}(\mathbf{R}), s \in C^{1,2}\left(R_{+} \times \mathbf{R}\right)$ and in addition, $K\left(x_{i}\right)=O\left(1+e^{2 x_{i}}\right)$, and $s\left(t, x_{i}\right)=$ $O\left(1+e^{x_{i}}\right)$, then the control law determined by $(5.5)$ is a Nash equilibrium for the costs (5.2) and an $\varepsilon$-Nash equilibrium for the costs (5.1) subject to full information for individual controls, where $\varepsilon=O\left(\frac{1}{\sqrt{n}}\right)$. 


\section{FURTHER WORK AND CONCLUSION}

To analyze solutions to the set of equations (5.3), (5.4), (5.6) and (5.7) one can in principle introduce a joint distribution function $F\left(t, x_{i}, p_{i}\right)$ for the process $\left(x_{i}, p_{i}\right)$ and express $z^{*}(t)$ in the form of an integral. Here $\left(x_{i}, p_{i}\right)$ is a Markov process and thus $F\left(t, x_{i}, p_{i}\right)$ is governed by a Fokker-Planck equation. Hence the evolution of the above equation system is described in terms of time $t$ and the infinite dimension state variable $\left(x_{i}, p_{i}, F(t, \cdot, \cdot)\right)$, which we shall call the generalized state variable. Possible approximation methods to the above equation system would be of great interest. This will be considered in future work.

In this paper we investigate stochastic power control subject to lognormal fading. Two different methods are considered: the global cost based centralized information control and the individual cost based decentralized control. In general, the global cost based approach emphasizes a certain coordination between individuals to achieve global optimality; for such large population systems the information used by a given individual exhibits a separation property in that its control law mainly depends upon (i) its own channel-power condition and (ii) a quantity measuring the nearly deterministic average effect of all the other users. It should be noted that in this centralized framework, each individual does not make an effort to optimize against this deterministic process, which distinguishes this case from the dynamic game theoretic scenario.

On the other hand, by virtue of the scaling nature of the cost function, we may consider approximating and then splitting the global cost function and thus obtain a decomposition into individual costs. This leads to a game theoretic framework. In such an individual cost based optimization setting, there is also a roughly deterministic process generated by the mass or collective. In contrast to the global cost case, each individual determines its control law by optimizing against the mass. Thus there is an intrinsic clash of interests between different users, but the individual and the mass can still reach stable behaviour under specific conditions.

\section{REFERENCES}

[1] T. Alpcan, T. Basar, R. Srikant, and E. Altman. CDMA uplink power control as a noncooperative game. Proc. 40th IEEE Conf. Decision Contr, Orlando, FL, pp.197202, Dec. 2001.

[2] A. Bensoussan. Stochastic Control of Partially Observable Systems. Cambridge University Press, 1992.

[3] R. Buche and H.J. Kushner. Control of mobile communications with time-varying channels in heavy traffic. IEEE Trans. Automat. Contr, vol.47, pp.992-1003, 2002.

[4] C.D. Charalambous and N. Menemenlis. Stochastic models for long-term multipath fading channels. Proc. 38th IEEE Conf. Decision Contr., Phoenix, AZ, pp.4947-4952, Dec. 1999.
[5] W.H. Fleming and H.M. Soner. Controlled Markov Processes and Viscosity Solutions, Springer-Verlag, 1993.

[6] M. Huang. Stochastic control for distributed systems with applications to wireless communications, Ph.D. Thesis, Dept. Electr. and Comput. Eng., McGill University, Montreal, Canada, June, 2003.

[7] M. Huang, P.E. Caines, C.D. Charalambous, and R.P. Malhamé. Stochastic power control for wireless systems: classical and viscosity solutions. Proc. 40th IEEE $C D C$, Orlando, FL., pp.1037-1042, Dec. 2001.

[8] M. Huang, P.E. Caines, and R.P. Malhamé. Uplink power adjustment in wireless communication systems: a stochastic control analysis. Under revision for IEEE Trans. Automat. Contr., 2003.

[9] M. Huang, P.E. Caines, and R.P. Malhamé. Degenerate stochastic control problems with exponential costs and weakly coupled dynamics: viscosity solutions and a maximum principle. Under revision for SIAM J. Contr. Optim., 2003.

[10] M. Huang, R.P. Malhamé, and P.E. Caines. Quality of service control for wireless systems: minimum power and minimum energy solutions. Proc. $A C C$, Anchorage, AK, pp.2424-2429, May, 2002.

[11] M. Huang, R.P. Malhamé, and P.E. Caines. Stochastic power control in wireless communication systems with an infinite horizon discounted cost. Proc. ACC., Denver, CO, pp.963-968, June, 2003.

[12] M. Huang, R.P. Malhamé, and P.E. Caines. Stochastic power control in wireless communication systems: analysis, approximate control algorithms and state aggregation. In Proc. 42nd CDC, Dec. 2003.

[13] QUALCOMM Inc. An overview of the application of Code Division Multiple Access (CDMA) to digital cellular systems and personal cellular networks. (Document no. EX60-10010), 1992.

[14] C.W. Sung and W.S. Wong. Mathematical aspects of the power control problem in mobile communication systems. AMS/IP Studies in Advanced Mathematics, vol.17, 2000.

[15] D.N.C. Tse and S.V. Hanly. Linear multiuser receivers: effective interference, effective bandwidth and user capacity. IEEE Trans. Infonn. Theory, vol.45, no.2, pp.641-657, 1999.

[16] S. Verdú and S. Shamai. Spectral efficiency of CDMA with random spreading. IEEE Trans. Inform. Theory, vol.45, no.2, pp.622-640, 1999.

[17] J. Zhang and E.K.P. Chong. CDMA systems in fading channels: admissibility, network capacity and power control. IEEE Trans. Inform. Theory, vol.46, no.3, pp.962-981, 2000. 